\title{
CLINICAL ISSUES
}

\section{Psychological and Physiological Stress: Impact on Preterm Birth}

\author{
Susan Gennaro, Mary Dawn Hennessy
}

Stress increases corticotropin-releasing hormone and may ultimately result in increased uterine contractility. Stress also increases cytokine production, which independently may lead to preterm birth or increase susceptibility to infection, thereby increasing the risk of preterm birth. Finally, stress may change health behaviors that lead to preterm birth. Research findings on the relationship between stress and preterm birth have been contradictory. In this article, the authors propose a model of the relationship between stress and preterm birth, evaluate the research on stress and pregnancy outcomes, and discuss the implications for nursing practice and research. JOGNN, 32, 668-675; 2003. DOI: $10.1177 / 0884217503257484$

Keywords: Cytokines-Pregnancy-Prematurity-Prostaglandins-Stress

Accepted: March 2003

Although it has been thought for centuries that increased stress leads to poorer health (Istevan, 1986), only recently has research rigorously demonstrated consistent relationships among increased stress, decreased immune status, and poorer health. In a landmark study, stressed healthy volunteers who were given nasal drops that contained either cold virus or normal saline had a dose-related increase in incidence of colds and flu when compared with nonstressed volunteers (Cohen, Tyrrell, \& Smith, 1991). Medical students experiencing examination stress had an increased incidence of self-reported infectious illness and a documented increase in Epstein-Barr antibodies, accompanied by decreases in interferon production (Glaser et al., 1987). Caregivers of Alzheimer patients who were more anxious and depressed (perhaps a sign of increased stress) had fewer circulating $\mathrm{T}$ cells and significantly more days of infectious illness when compared with less anxious and less depressed caregivers (Kiecolt-Glaser, Dura, Speicher, Trask, \& Glaser, 1991).

As the relationship between increased stress, poorer immune status, and poorer overall health has become better established, investigations have begun into whether stress increases the likelihood of other adverse health outcomes, such as preterm birth. Stress may affect preterm birth either through an increased susceptibility to infections (Wadhwa et al., 2001) or as a result of immune changes that result from stress even in the absence of infection (Ruiz \& Pearson, 1999). However, not all women who experience high levels of stress or who experience intrapartum infections deliver preterm infants, so further work is needed to better determine the mechanism underlying preterm labor and delivery. The purpose of this article is to provide an overview of the literature regarding psychological and physiological stress and preterm birth, to propose physiological mechanisms whereby stress may increase the risk of preterm birth, and, given this model, to discuss implications for nursing practice and research.

\section{Psychological Stress and Preterm Birth}

\section{Challenges in Measuring Stress}

The relationship between stress and birth outcomes is unclear, perhaps in part because researchers have been inconsistent in defining and measuring stress. Conceptual and methodological inconsistencies make it difficult to identify exactly what kinds 
of stress, experienced at what points in pregnancy, and by which women, are most likely to lead to poor pregnancy outcomes such as preterm birth.

Preterm birth has multiple causes, making research on stress and preterm birth even more difficult to evaluate. Preterm birth that occurs after 34 weeks of completed gestational age is more likely to be associated with placental and circulatory problems (such as pregnancyinduced hypertension, diabetes, etc.) than is earlier preterm delivery, in which infection and stress are key risk factors (Goldenberg \& Rouse, 1998). When the impact of stress is evaluated, it is also important to consider whether preterm delivery is spontaneous or medically indicated.

\section{$\Delta$ s the relationship between increased} stress, poorer immune status, and poorer overall health has become better established, investigations have begun into whether stress increases the likelihood of other adverse health outcomes, such as preterm birth.

Findings regarding the link between stress and preterm birth also differ based on how stress is conceptualized. Stress has been measured by negative life events (Mutale, Creed, Maresh, \& Hunt, 1991; Whitehead, Hill, Brogan, \& Blackmore-Prince, 2002; Zuckerman, Amaro, Bauchner, \& Cabral, 1989) and has also been conceptualized as experiencing daily hassles (DaCosta, Brender, \& Larouche, 1998; Hobel, Dunkel-Schetter, Roesch, Castro, \& Arora, 1999; Wadhwa, Sandman, Porto, DunkelSchetter, \& Garite, 1993), psychological distress (often measured by anxiety or depression) (Hobel et al., 1999; Killingsworth Rini, Dunkel-Schetter, Sandman, \& Wadhwa, 1999; Wadhwa et al., 1993), and by perceived stress (Lobel, Dunkel-Schetter, \& Scrimshaw, 1992; Sable \& Wilkinson, 2000).

For example, Hedegaard, Henriksen, Secher, Hatch, and Sabroe (1996) studied stressful life events in 5,873 Danish women and found that experiencing negative life events did not increase the risk of poor birth outcomes. When the women's appraisal of the stress involved in these life events was examined, the women who perceived life events to be highly stressful had a 1.76 times greater risk of preterm delivery than did the women who perceived less stress.

Life events such as changes in residence and changes in employment might be more likely to occur during preg- nancy. Lobel (1994) suggested and Hedegaard et al. (1996) documented that it is more appropriate to ask women what they experience as stressful than to assume that events that might be stressful at other points in time are necessarily perceived as negative or stressful during pregnancy. For example, changes in residence and employment might be viewed more positively during pregnancy than at other times. Pregnant women generally have a lower incidence of negative life events than other groups (Istevan, 1986), so it is not clear that life events are the best measure of stress during pregnancy. Questions have also been raised as to whether there is a threshold of a particular number of life events, beyond which women have an increased risk of preterm delivery (Whitehead et al., 2002).

Many researchers have measured stress perception (Copper et al., 1996; Hobel et al., 1999; Lobel et al., 1992; Sable \& Wilkinson, 2000) rather than simply counting life events (Mutale et al., 1991; Whitehead et al., 2002; Zuckerman et al., 1989). Some researchers combined measures of life events and measures of perceived stress (Lobel et al., 1992; Sable \& Wilkinson, 2000; Sandman et al., 1994; Wadhwa et al., 1993; Wadhwa, DunkelSchetter, Chicz-DeMet, Porto, \& Sandman, 1996). Researchers who combined multiple measures of stress have generally found that women who experienced increased stress during pregnancy were more likely to deliver prematurely (Copper et al., 1996; Hedegaard et al., 1996; Lobel et al., 1992).

When measures of psychological distress (e.g., anxiety or depression) were used as surrogates for stress, they were linked to preterm birth in two studies (Davids \& DeVault, 1962; Davids, DeVault, \& Talmadge, 1961), whereas in a third study, increased anxiety was not related to preterm birth (Perkin, Bland, Peacock, \& Anderson, 1993). Some differences in these results may be explained by the type and timing of measurements and by sample sizes.

When both anxiety and stress were measured, a difference in their impact was found. Wadhwa et al. (1996) found that with every point increase in anxiety, there was an increase in preterm birth, but increases in stress affected birth weight, not gestational age. Pagel, Smilkstein, Regen, and Montano (1990) also found that increased anxiety was related to preterm delivery, but increases in stress (measured as increased life events) were related to lower birth weight but not to earlier delivery. Although stress might lead to a psychological response such as increased anxiety, stress and anxiety are not synonymous. Anxiety may be one psychological response to stress, but stress may result in other emotional responses, such as depression or anger. Therefore, women may experience increased stress without an increase in anxiety.

Asking women directly about perceived stress may be a more accurate way to identify acute stress than using 
measures of psychological distress such as anxiety, which may be a stress response. Perceived stress may also be a more accurate measure of stress than are life events because perceived stress accounts for the individual's response to the situation, rather than simply measuring the occurrence of stressful events.

\section{Timing of Stress Measures in Pregnancy}

Different timing of stress measurement in pregnancy has produced inconsistent findings. Stress has been measured in the 2nd trimester (Copper et al., 1996; Hobel et al., 1999; Yali \& Lobel, 1999), the 3rd trimester (Copper et al., 1996; Hobel et al., 1999; Sandman, Wadhwa, Chicz-DeMet, Dunkel-Schetter, \& Porto, 1997; Sandman et al., 1994; Wadhwa et al., 1993; Wadhwa et al., 1996), and in the postpartum period (Mutale et al., 1991; Sable \& Wilkinson, 2000; Sandman et al., 1997; Whitehead et al., 2002). Longitudinal studies are more comprehensive in capturing the degree of stress over time (Hedegaard et al., 1996; Hobel et al., 1999; Lobel et al., 1992), whereas cross-sectional studies are limited to measurement at one moment in time (Mutale et al., 1991; Sable \& Wilkinson, 2000; Sandman et al., 1994; Whitehead et al., 2002; Yali \& Lobel, 1999).

When researchers asked about stress retrospectively (Mutale et al., 1991; Sable \& Wilkinson, 2000; Whitehead et al., 2002), women seeking meaning for a preterm birth or other poor pregnancy outcome may have been more likely to recall their pregnancies as stressful. Studies with prospective research designs avoid this problem of

\section{Conceptual and methodoaical Ionceptual and methodological inconsistencies make it difficult to identify exactly what kinds of stress, experienced at what point in pregnancy, and by which women, are most likely to lead to preterm birth.}

recall bias (Copper et al., 1996; Hobel et al., 1999; Lobel et al., 1992; Sandman et al., 1994; Sandman et al., 1997; Wadhwa et al., 1993; Wadhwa et al., 1996; Zuckerman et al., 1989). Despite using many different measures for stress, researchers still generally found links between increased stress and poor pregnancy outcomes.

It is not known if there is a critical point in pregnancy when experiencing acute stress is more detrimental to pregnancy outcomes than at other times or if chronic or acute stress leads to preterm delivery. In prospective studies in which stress was measured at more than one time point, increased stress over time was related to poor preg- nancy outcomes (DaCosta et al., 1998) and specifically to preterm birth (Hedegaard et al., 1996); however, high levels of stress in early pregnancy were not related to preterm birth (Hedegaard et al., 1996), lending support to the idea that a more constant experience of stress is necessary for preterm birth to occur.

\section{Sample Differences and Other Confounding Factors}

Other methodological issues that make it difficult to be certain about the role of stress in a study or to generalize across studies include weak research designs, inadequate sample size and quality, differing definitions of outcome variables, and lack of control of confounding variables. For example, other risk factors for preterm birth, such as smoking, poor nutrition, and drug use, should be adequately measured so that the true contribution of stress itself can be evaluated.

When ethnically and socioeconomically diverse samples were examined, stress was linked to preterm birth (Copper et al., 1996; Hobel et al., 1999; Sable \& Wilkinson, 2000). Because there is a higher incidence of preterm birth among Hispanic and African American populations than among White women (Agency for Healthcare Research and Quality [AHRQ], 2000), some researchers have proposed that demographic stressors such as racism may lead to preterm birth (Rich-Edwards et al., 2001). Others have studied stress and preterm birth only in homogeneous samples, producing findings representative of one race or a single parity status, socioeconomic class, educational background, or partner status (Lobel et al., 1992; Rothberg \& Lits, 1991; Sandman et al., 1994; Wadhwa et al., 1993; Wadhwa et al., 1996; Whitehead et al., 2002; Yali \& Lobel, 1999; Zuckerman et al., 1989).

In some studies, sample sizes were small (less than 200 ). Because preterm birth occurs in only about $7 \%$ of births, small sample sizes sometimes resulted in a relatively small number of women who experienced preterm birth (Lobel et al., 1992; Mutale et al., 1991; Sandman et al., 1994; Wadhwa et al., 1993; Wadhwa et al., 1996; Yali \& Lobel, 1999). However, there are a number of studies with larger samples, and the relationship between stress and preterm birth has been documented more reliably with samples of more than 500 women (Copper et al., 1996; Hobel et al., 1999; Sable \& Wilkinson, 2000; Whitehead et al., 2002).

\section{Physiological Reactions to Stress}

\section{Stress and CRH Release}

The exact mechanisms of how labor begins, whether at term or preterm, are not clear. One of the most important questions in obstetrics that remains to be answered is why some women who start to labor prematurely progress to 
deliver preterm infants, whereas other women do not. As our understanding of the relationship between psychological stress and preterm birth increases, there has also been exciting research linking physiological changes of stress to birth outcomes.

Stress may increase the likelihood of preterm birth by increasing corticotropin-releasing hormone (CRH), a neuropeptide synthesized primarily in the paraventricular nucleus of the hypothalamus (McLean et al., 1995). During pregnancy, CRH also is synthesized by the placenta and membranes and increases exponentially in the final weeks before a full-term delivery. As CRH rises with the advancing pregnancy, there is a fall in concentrations of CRH-binding protein, leading to an increase in the levels of CRH available for the body to use. CRH release leads to increased adrenocorticotropin hormone and increased glucocorticoids, which in turn up-regulate $\mathrm{CRH}$ in the placenta and stimulate prostaglandin production and labor (Challis, 2000).

$\mathrm{CRH}$ was found to be present at higher levels in women who went on to deliver preterm than in women who had normal, full-term pregnancies (Warren, Patrick, \& Goland, 1992), supporting the link between stress and preterm delivery. For example, Wadhwa, Porto, Garite, Chicz-DeMet, and Sandman (1998) studied 63 pregnant women and found that elevated maternal CRH levels measured between 28 and 30 weeks significantly and negatively predicted gestational length. In a larger study, McLean et al. (1995) studied 485 pregnant women and found that CRH levels at 16-20 weeks of pregnancy accurately predicted which women would deliver preterm, at term, or postterm.

\section{Cytokines in Pregnancy}

A number of studies in nonpregnant subjects have documented increased cytokine production with increased stress (Maes, Christophe, Bosmans, Lin, \& Neels, 2000; Maes et al., 1998a, 1999). Stress increases proinflammatory cytokine production (Chouros, 1995; Turnbull \& Rivier, 1999), which leads to increased prostaglandin production, increased uterine contractility, and preterm labor. Increased stress may lead to increased susceptibility to infection. Infections also increase cytokine production and can lead to increased prostaglandin production and preterm labor (see Figure 1). In a negative feedback loop, increases in stress hormones such as cortisol signal the body to decrease production of proinflammatory cytokines and hormones, in an effort to maintain homeostasis (Chouros, 1995; Turnbull and Rivier, 1999).

Cytokines are small molecular-weight proteins that act as messengers between immune cells. Some cytokines promote cellular immunity, helping the body to distinguish self from non-self and self from altered self. They help the body to destroy mutagenic cells and foreign cells such as viruses and parasites. Other cytokines support humoral

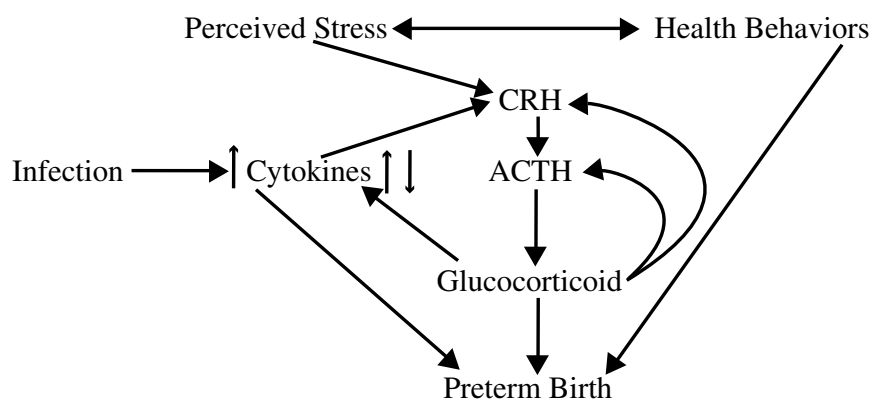

FIGURE 1

Hormonal Response to Stressors Leading to Preterm Birth

$\mathrm{CRH}=$ corticotropin-releasing hormone; $\mathrm{ACTH}=$ adrenocorticotropin hormone.

immunity, working to destroy foreign cells, including bacteria, by signaling the production of immunoglobulins.

Whereas both kinds of cytokines are necessary and work together (controlling the response of one another), humoral immunity is favored over cellular immunity in pregnancy. Cellular immunity is somewhat diminished, to protect fetal cells from destruction by the mother.

Although lowered cellular immunity places pregnant women at greater risk for some kinds of diseases (including tuberculosis and malaria), the overall benefit is positive because the fetus is protected. After the implantation phase of pregnancy, there is an increase in the production of cytokines that promote humoral immunity (Wegmann, Lin, Guilbert, \& Mosmann, 1993). Cytokines that favor fetal survival and growth include IL-3, GN-CSF, TGF- $\beta$, IL-4, and IL-10.

\section{Cytokines and Prostaglandin}

The cytokines that favor cellular immunity, although decreased in normal pregnancy, may facilitate preterm labor through their action on prostaglandin production. Prostaglandins mediate labor onset by ripening the cervix, increasing calcium levels in the cytoplasm of smooth muscle, and forming the gap junction.

Although there are more than 60 cytokines, interleukin-1 (IL-1), IL-6, and TNF- $\alpha$ have most consistently been found to be elevated in the serum, vaginal tract, and amniotic fluid of women experiencing preterm delivery. Each of these three cytokines plays a role in increasing prostaglandin synthesis (Lockwood, 1994). IL-1 stimulates prostaglandin production, and also acts to augment $\mathrm{T}$ and $\mathrm{B}$ cells and neutrophils, to induce fever and to potentiate the hepatic acute phase response in association with IL-6 (Dudley, 1992). IL-6 stimulates the production of prostaglandins and activates T cells (Heinrich, Castell, \& Andus, 1990). Most cells can produce IL-6, and IL-1 and TNF- $\alpha$ are secreted primarily by lymphocytes and macrophages. TNF- $\alpha$ enhances fibrin deposition and stimulates prostaglandin biosynthesis (Heinrich et al., 1990). 
Cytokines like IL-1, IL-6, and TNF- $\alpha$ that can compromise pregnancy are found only in small amounts during normal pregnancies. For example, Opsojon and colleagues (1993) found only minimal amounts of TNF- $\alpha$, IL-amniotic 1, and IL-6 in amniotic fluid during the 1st trimester of pregnancy, but amniotic fluid IL-6 was present at term and increased dramatically with the onset of labor, as did TNF and IL-1. Dudley, Hunter, Mitchell, and Varner (1994) and Turhan, Karabulut, and Adam (2000) found that women in active labor had increased levels of IL-6 compared to women who were not in labor.

Elevated cytokine levels have been found in the amniotic fluid of women with intrauterine infection and those who went on to deliver prematurely. Higher amniotic fluid levels of IL-6 were observed in women in preterm labor with intra-amniotic infection than in women in preterm labor without infection (Romero, Avila, Santhanam, \& Sehgal, 1990). Negishi and colleagues (1996) found that an amniotic fluid Il-6 level of more than 600 picograms per milliliter $(\mathrm{pg} / \mathrm{ml})$ had a $100 \%$ positive predictive value and a $92 \%$ negative predictive value in detecting histological chorioamnionitis in women who experienced preterm delivery.

Amniotic fluid IL-6 levels were elevated during the early 2 nd trimester in women with a greater risk of preterm delivery (Ghidini et al., 1997). Hillier et al. (1993) found a strong association between preterm delivery and high amniotic fluid levels of IL-6. Il-6 concentrations greater than $1,500 \mathrm{pg} / \mathrm{ml}$ were detected in $88 \%$ of the women who delivered at or before 34 weeks gestation.

\section{Despite the lack of agreement on the best way to measure stress, the link between perceived stress and preterm birth is strong enough that nurses who care for pregnant women should ask regularly about the amount of stress in women's lives.}

Findings of elevated IL-6 in amniotic fluid in women with premature delivery have also been documented by a number of other researchers around the world (Saito, Kasahara, Kato, Ishihara, \& Ichijo, 1993; Steinborn, Kuhnert, \& Halberstadt, 1996).

Cytokines are increased in maternal serum and in the vaginal tract, as well as in the amniotic fluid of women with threatened preterm delivery. Inglis et al. (1994) found that the presence of IL- 6 and TNF- $\alpha$ in the lower genital tract of women with threatened preterm labor was associated with a greater than threefold increase in risk of preterm delivery. Likewise, Murtha and colleagues (1996) found that maternal serum IL-6 levels were elevated in women with premature rupture of membranes and served as a sensitive $(81 \%)$ and specific $(99 \%)$ indicator of infection. Serum IL-6 concentrations higher than $8 \mathrm{pg} / \mathrm{ml}$ at 22-34 weeks gestation were predictive of preterm delivery (Murtha, Greig, Jimmerson, \& Herbert, 1998). Greig et al. (1997) found maternal serum IL-6 levels were highest in women in preterm labor with chorioamnionitis but were also elevated in women in preterm labor with no chorioamnionitis, arguing for the importance of other factors, such as stress, as contributors to preterm delivery.

\section{Stress and Health Behaviors}

Stress may change health behaviors such as smoking, eating, and sleeping. Increased smoking and poor nutrition are both implicated in preterm delivery (Brooke, Anderson, Bland, Peacock, \& Stewart, 1989; Cliver et al., 1992; Cnattingius, Granath, Petersson, \& Harlow, 1999). The stress of poverty may lead to increases in these unhealthy behaviors, suggesting one reason why lowincome women are at risk for preterm birth (Kramer et al., 2001).

\section{Implications for Research and Practice}

Only $50 \%$ of women who experience preterm labor go on to deliver prematurely (AHRQ, 2000). Despite the promise of the physiological studies described above, at present there are no physiological or psychological measures that can predict which individuals will deliver prematurely. It is likely that a combination of factors, including lifestyle choices, stress, and infection, contribute to preterm labor and preterm delivery.

Despite the lack of agreement on the best way to measure stress, the link between perceived stress and preterm birth is now strong enough that nurses who care for pregnant women should ask regularly about the amount of stress in women's lives. Nurses can help pregnant women identify health behaviors that can be modified to decrease the likelihood of preterm birth, including decreasing stress, ensuring adequate nutrition, and avoiding smoking. This is particularly important for women who are at risk for preterm labor before 34 weeks gestation.

Further research on the biophysiological roots of preterm labor and delivery is necessary to develop more sensitive ways to identify those women at greatest risk for preterm delivery. Additionally, it is imperative to determine the relative strength of a number of important contributors to preterm labor. Although anxiety has been considered a product of stress, research is needed to test these conceptual links between stress and psychological responses such as anxiety. Large prospective studies are 
needed to adequately evaluate the relationships among stress, anxiety, and preterm birth.

Our lack of knowledge of which women are most likely to experience preterm labor and which women are likely to go on to deliver prematurely is frustrating. No doubt, susceptibility to preterm delivery is affected by environmental and genetic contributions as well as an interaction of psychological and physiological responses to stress. Nurses are in a unique position to develop culturally competent models of care that give women the information they need to modify risk factors that are amenable to change. As we better understand the psychological and physiological factors that underlie preterm birth, nurses will be poised to develop new interventions that help women reduce risk factors and achieve positive birth outcomes.

\section{REFERENCES}

Agency for Healthcare Research and Quality. (2000). Management of preterm labor (Evidence Report, Technology Assessment No.18, Publication 01-E021). Rockville, MD: Author.

Brooke, O., Anderson, H., Bland, J., Peacock, J., \& Stewart, C. (1989). Effects on birth weight of smoking alcohol, caffeine, socioeconomic factors and psychosocial stress. British Medical Journal, 298, 795-801.

Challis, J. R. G. (2000). Mechanism of parturition and preterm labor. Obstetrical and Gynecological Survey, 10, 650660.

Chouros, G. P. (1995). The hypothalamic-pituitary-adrenal axis and immune-mediated inflammation. New England Journal of Medicine, 332, 1351-1362.

Cliver, S., Goldenberg, R., Cutter, G., Hoffman, H., Copper, R., Gotlieb, S., et al. (1992). The relationships among psychosocial profile, maternal size, and smoking in predicting fetal growth retardation. Obstetrics and Gynecology, 80, 262-267.

Cnattingius, S., Granath, F., Petersson, G., \& Harlow, B. (1999). The influence of gestational age and smoking habits on the risk of subsequent preterm deliveries. New England Journal of Medicine, 341, 943-948.

Cohen, S., Tyrrell, D., \& Smith, A. (1991). Psychological stress and susceptibility to the common cold. New England Journal of Medicine, 325, 606-612.

Copper, R., Goldenberg, R., Das, A., Elder, N., Swain, M., Norman, G., et al. (1996). The preterm prediction study: Maternal stress is associated with spontaneous preterm birth at less than thirty-five weeks gestation. American Journal Obstetrics and Gynecology, 175, 1286-1292.

DaCosta, D., Brender, W., \& Larouche, J. (1998). A prospective study of the impact of psychosocial and lifestyle variables on pregnancy complications. Journal of Psychosomatic Obstetrics and Gynaecology, 37, 28-37.

Davids, A., \& DeVault, S. (1962). Maternal anxiety during pregnancy and childbirth abnormalities. Psychosomatic Medicine, 24, 464-470.
Davids, A., DeVault, S., \& Talmadge, M. (1961). Anxiety, pregnancy, and childbirth abnormalities. Journal of Consulting Psychology, 25, 74-77.

Dudley, D. (1992). The immune system in health and disease. Balliere's Clinical Obstetrics and Gynecology, 6, 393417.

Dudley, D., Hunter, C., Mitchell, M., \& Varner, M. (1994). Clinical value of amniotic fluid interleukin-6 determinations in the management of preterm labour. British Journal of Obstetrics and Gynaecology, 101, 592-597.

Ghidini, A., Jenkins, C., Spong, C., Pezzullo, J., Salafia, C., \& Eglington, G. (1997). Elevated amniotic fluid interleukin6 levels during the early second trimester are associated with greater risk of subsequent preterm delivery. American Journal of Reproductive Immunology, 37, 227-231.

Glaser, R., Rice, J., Sheridan, J., Fertel, R., Stout, J., Speicher, C. E., et al. (1987). Stress-related immune suppression: Health implications. Brain, Behavior, and Immunity, 1, 720.

Goldenberg, R., \& Rouse, D. (1998). Prevention of premature birth. New England Journal of Medicine, 339, 313-320.

Greig, P., Murtha, A., Jimmerson, C., Herbert, W., RoitmanHohnson, B., \& Allen, J. (1997). Maternal serum interleukin-6 during pregnancy and during term and preterm labor. Obstetrics and Gynecology, 90, 465-469.

Hedegaard, M., Henriksen, T. B., Secher, N. J., Hatch, M., \& Sabroe, S. (1996). Do stressful life events affect duration of gestation and risk of preterm delivery? Epidemiology, 7, 339-345.

Heinrich, P., Castell, J., \& Andus, T. (1990). Interleukin 6 and the acute phase response. Biochemical Journal, 265, 621636.

Hillier, S., Witkin, S., Krohn, M., Watts, D., Kiviat, N., \& Eschenbach, D. (1993). The relationship of amniotic fluid cytokines and preterm delivery, amniotic fluid infection, histologic chorioamnionitis and chorioamnion infection. Obstetrics \& Gynecology, 81, 941-948.

Hobel, C., Dunkel-Schetter, C., Roesch, S., Castro, L., \& Arora, C. (1999). Maternal plasma corticotropin-releasing hormone associated with stress at 20 weeks gestation in pregnancies ending in preterm delivery. American Journal of Obstetrics and Gynecology, 180, S257-S263.

Inglis, S., Jeremias, J., Kuno, K., Lesale, K., Peeper, Q., Chervenak, F., et al. (1994). Detection of tumor necrosis factor$\alpha$, interleukin- 6 , and fetal fibronectin in the lower genital tract during pregnancy: Relation to outcome. American Journal of Obstetrics and Gynecology, 171, 5-10.

Istevan, J. (1986). Stress, anxiety, and birth outcomes: A critical review of the evidence. Psychological Bulletin, 100, 331348.

Kiecolt-Glaser, J., Dura, J., Speicher, C., Trask, J., \& Glaser, R. (1991). Spousal caregivers of dementia victims: Longitudinal changes in immunity and health. Psychosomatic Medicine, 53, 345-362.

Killingsworth Rini, C., Dunkel-Schetter, C., Sandman, C., \& Wadhwa, P. (1999). Psychological adaptation and birth outcomes: The role of personal resources, stress and sociocultural context in pregnancy. Health Psychology, 18, 333-345. 
Kramer, M., Goulet, L., Lydon, J., Seguin, L., McNamara, H., Dassa, C., et al. (2001). Socio-economic disparities in preterm birth: Causal pathways and mechanisms. Paediatric and Perinatal Epidemiology, 15, 104-123.

Lobel, M. (1994). Conceptualizations, measurement and effects of prenatal maternal stress on birth outcomes. Journal of Behavioral Medicine, 17, 225-273.

Lobel, M., Dunkel-Schetter, C., \& Scrimshaw, S. (1992). Prenatal maternal stress and prematurity: A prospective study of socioeconomically disadvantaged women. Health Psychology, 11, 32-40.

Lockwood, C. (1994). Recent advances in elucidating the pathogenesis of preterm delivery, the detection of patients at risk, and preventative therapies. Current Opinion in Obstetrics and Gynecology, 6, 7-18.

Maes, M., Christophe, A., Bosmans, E., Lin, A., \& Neels, H. (2000). In humans, serum polyunsaturated fatty acid levels predict the response of proinflammatory cytokines to psychologic stress. Biologic Psychiatry, 47, 910-920.

Maes, M., Song, C., Lin, A., De Jongh, R., Van Gastel, A., Kenis, G., et al. (1998a). The effects of psychological stress on humans: Increased production of pro-inflammatory cytokines and a Th 1-like response in stress-induced anxiety. Cytokine, 10, 313-318.

Maes, M., Janca, A., Scharpe, S., Smith, R. S., Song, C., \& Lin, A. (1998b). Immune and clinical correlates of psychological stress-induced production of interferon-gamma and IL-10 in humans. In N. P. Potnikoff, R. E. Faith, A. J. Murgo, \& R. A. Good. (Eds.), Cytokines, stress and immunity (pp. 39-50). Boca Raton, FL: Raven Press.

McLean, M., Bisits, A., Davies, J., Woods, R., Lowry, P., \& Smith, R. (1995). A placental clock controlling the length of human pregnancy. Nature Medicine, 1, 460-463.

Murtha, A., Greig, P., Jimmerson, C., \& Herbert, W. (1998). Maternal serum interleukin- 6 concentration as a marker for impending delivery. Obstetrics \& Gynecology, 91, 161-164.

Murtha, A., Greig, P., Jimmerson, C., Roitman-Hohnson, B., Allen, J., \& Herbert, W. (1996). Maternal serum interleukin- 6 concentrations in patients with preterm premature rupture of membranes and evidence of infection. American Journal Obstetrics and Gynecology, 175, 966969.

Mutale, T., Creed, F., Maresh, M., \& Hunt, L. (1991). Life events and low birthweight-analysis by infants preterm and small for gestational age. British Journal of Obstetrics and Gynaecology, 98, 166-172.

Negishi, H., Yamada, H., Mikuni, M., Kishida, T., Okuyama, K., Sagawa, T., et al. (1996). Correlation between cytokine levels of amniotic fluid and histological chorioamnionitis in preterm delivery. Journal of Perinatal Medicine, 24, 633-639.

Opsojon, S., Wathen, N., Tingulstad, S., Wiedswang, G., Sundan, A., Waage, A., et al. (1993). Tumor necrosis factor, interleukin-1 and interleukin-6 in normal human pregnancy. American Journal of Obstetrics and Gynecology, 169, 397-404.

Pagel, M., Smilkstein, G., Regen, H., \& Montano, D. (1990). Psychosocial influences on newborn outcomes: A con- trolled prospective study. Social Science and Medicine, 30, 597-604.

Perkin, M. R., Bland, J. M., Peacock, J. L., \& Anderson, H. R. (1993). The effect of anxiety and depression during pregnancy on obstetric complications. British Journal of Obstetrics and Gynaecology, 100, 629-634.

Rich-Edwards, J., Krieger, N., Majzoub, J., Zierler, S., Lieberman, E., \& Gillman, M. (2001). Maternal experiences of racism and violence as predictors of preterm birth: Rationale and study design. Paediatric and Perinatal Epidemiology, 15, S124-S135.

Romero, R., Avila, C., Santhanam, U., \& Sehgal, P. (1990). Amniotic fluid interleukin 6 in preterm labor. Association with infection. Journal of Clinical Investigation, 85, 1392-1400.

Rothberg, A. D., \& Lits, B. (1991). Psychosocial support for maternal stress during pregnancy: Effect on birth weight. American Journal of Obstetrics and Gynecology, 165, 403-407.

Ruiz, R. J., \& Pearson, A. J. (1999). Psychoneuroimmunology and preterm birth. A holistic model for obstetrical nursing practice and research. Maternal Child Nursing, 24, 230-235.

Sable, M., \& Wilkinson, D. (2000). Impact of perceived stress, major life events and pregnancy attitudes on low birth weight. Family Planning Perspectives, 32, 288-294.

Saito, S., Kasahara, T., Kato, Y., Ishihara, Y., \& Ichijo, M. (1993). Elevation of amniotic fluid interleukin 6 (IL-6), IL-8, and granulocyte colony stimulating factor (G-CSF) in term and preterm parturition. Cytokine, 5, 81-88.

Sandman, C. A., Wadhwa, P., Dunkel-Schetter, C., ChiczDeMet, A., Belman, J., Porto, M., et al. (1994). Psychobiological influence of stress and HPA regulation on the human fetus and infant birth outcomes. Annals of the New York Academy of Sciences, 739, 198-210.

Sandman, C. A., Wadhwa, P. D., Chicz-DeMet, A., DunkelSchetter, C., \& Porto M. (1997). Maternal stress, HPA activity, and fetal/infant outcome. Annals of the New York Academy of Sciences, 814, 266-275.

Steinborn, A., Kuhnert, M., \& Halberstadt, E. (1996). Immunmodulating cytokines induce term and preterm parturition. Journal of Perinatal Medicine, 24, 381-390.

Turhan, N., Karabulut, A., \& Adam, B. (2000). Maternal serum interleukin 6 levels in preterm labor: Prediction of admission-to-delivery interval. Journal of Perinatal Medicine, 28, 133-139.

Turnbull, A., \& Rivier, C. (1999). Regulation of the hypothalamic-pituitary-adrenal axis by cytokines: Actions and mechanisms of action. Physiological Reviews, 79, 1-71.

Wadhwa, P., Dunkel-Schetter, C., Chicz-DeMet, A., Porto, M., \& Sandman, C. (1996). Prenatal psychosocial factor and the neuroendocrine axis in human pregnancy. Psychosomatic Medicine, 58, 432-446.

Wadhwa, P., Porto, M., Garite, T., Chicz-DeMet, A., \& Sandman, C. (1998). Maternal corticotropin-releasing hormone levels in the early third trimester predict level of gestation in human pregnancy. American Journal of Obstetrics and Gynecology, 179, 1079-1085.

Wadhwa, P., Sandman, C., Porto, M., Dunkel-Schetter, C., \& Garite, T. (1993). The association between prenatal stress 
and infant birth weight and gestational age at birth: A prospective investigation. American Journal of Obstetrics \& Gynecology, 169, 858-865.

Wadhwa, P. D., Culhane, J. F., Rauh, V., Barve, S. S., Hogan, V., Sandman, C. A., et al. (2001). Stresses, infection and preterm birth: A biobehavioural perspective. Paediatric and Perinatal Epidemiology, 15, 17-29.

Warren, W., Patrick, S., \& Goland, R. (1992). Elevated maternal plasma corticotropin-releasing hormone levels in pregnancies complicated by preterm labor. American Journal of Obstetrics and Gynecology, 166, 1198-1207.

Wegmann, T., Lin, H., Guilbert, L., \& Mosmann, T. (1993). Bidirectional cytokine interactions in the maternal-fetal relationship: Is successful pregnancy a $\mathrm{T}_{\mathrm{H}} 2$ phenomenon? Immunology Today, 14, 353-356.

Whitehead, N., Hill, H., Brogan, D., \& Blackmore-Prince, C. (2002). Exploration of threshold analysis in the relation between stressful life events and preterm delivery. American Journal of Epidemiology, 155, 177-124.

Yali, A. M., \& Lobel, M. (1999). Coping and stress in pregnancy: An investigation of medically high-risk women. Jour- nal of Psychosomatic Obstetrics and Gynecology, 20, 3952.

Zuckerman, B., Amaro, H., Bauchner, H., \& Cabral, H. (1989). Depressive symptoms during pregnancy: Relationship to poor health behaviors. American Journal of Obstetrics and Gynecology, 160, 1107-1011.

Susan Gennaro, DSN, RN, FAAN, is a professor in the School of Nursing, University of Pennsylvania, Philadelphia.

Mary Dawn Hennessy, MSN, RN, is a doctoral student, International Center of Research for Women, Children and Families, University of Pennsylvania, School of Nursing, Philadelphia.

Address for correspondence: Susan Gennaro, DSN, RN, FAAN, University of Pennsylvania, School of Nursing, 420 Guardian Drive, Philadelphia, PA 19104-6096. E-mail: gennaro@nursing.upenn.edu. 